\title{
Response to influenza vaccination in immunocompromised children with rheumatic disease: a prospective cohort study
}

Lotte Jensen ${ }^{1,2^{*}}$, Susan Nielsen ${ }^{1}$, Anne Estmann Christensen ${ }^{3}$, Freddy Karup Pedersen ${ }^{1}$, Ramona Trebbien ${ }^{4}$, Thea Kølsen Fischer ${ }^{4}$, Susanne Rosthøj ${ }^{5}$, Peter Toftedal ${ }^{3}$, Anna-Helene Bohr ${ }^{6}$, Peder Skov Wehner ${ }^{3}$ and Anja Poulsen ${ }^{1}$

\begin{abstract}
Background: Prevention of illness due to infection by influenza viruses is important for children with rheumatic diseases. Biological disease modifying antirheumatic drugs have become increasingly important in the treatment of juvenile idiopathic arthritis, and combinations of immunosuppressive drugs are used for the treatment of systemic disorders, which increase the risk of secondary immunodeficiency. Therefore, we investigated whether children with rheumatic disease can mount a protective antibody response after influenza immunization.

Methods: The prospective multicentre cohort study was conducted in Denmark during the influenza season 20152016. Children with rheumatic disease aged six months to 19 years were eligible. Controls were immunologically healthy children. A blood sample was collected before and after vaccination and analysed by haemagglutination inhibition (HI) assay for the 2015-2016 influenza vaccine-strains. In case of flu-like symptoms the child was tested for influenza. For statistical analyses the patients were grouped according to medical treatment or disease.

Results: A total of 226 patients and 15 controls were enrolled. No differences were found for the increase of antibodies from pre-vaccine to post-vaccine between the groups in our primary analyses: A/Cal H1N1pdm09 ( $p=$ $0.28)$, A/Swi H3N2 $(p=0.15)$ and B/Phu Yamagata $(p=0.08)$. Only when combining patients across groups a lower increase in antibodies was found compared to controls. Among all patients the pre-vaccine rates for seroprotection using the HI-titer cut-off $\geq 40$ were $93.1-97.0 \%$ for all three strains. For seroprotection using the HI-titer cut-off $\geq$ 110 the pre-vaccine rates for all patients were $14.9-43.6 \%$ for all three strains and an increase in the proportions of patients being seroprotected after vaccination was found for A/Cal H1N1pdm09 and A/Swi H3N2. None of the children with flu-like symptoms tested positive for the vaccine strains.

(Continued on next page)
\end{abstract}

\footnotetext{
* Correspondence: lotte.jensen@regionh.dk

${ }^{1}$ The Department of Paediatrics and Adolescent Medicine, Juliane Marie

Centre, Rigshospitalet, Copenhagen University Hospital, Copenhagen,

Denmark

${ }^{2}$ Faculty of Health and Medical Sciences, University of Copenhagen,

Copenhagen, Denmark

Full list of author information is available at the end of the article
}

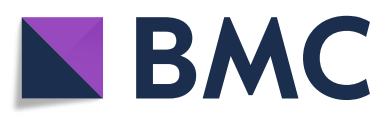

(c) The Author(s). 2021, corrected publication 2021. Open Access This article is licensed under a Creative Commons Attribution 4.0 International License, which permits use, sharing, adaptation, distribution and reproduction in any medium or format, as long as you give appropriate credit to the original author(s) and the source, provide a link to the Creative Commons licence, and indicate if changes were made. The images or other third party material in this article are included in the article's Creative Commons licence, unless indicated otherwise in a credit line to the material. If material is not included in the article's Creative Commons licence and your intended use is not permitted by statutory regulation or exceeds the permitted use, you will need to obtain permission directly from the copyright holder. To view a copy of this licence, visit http://creativecommons.org/ licenses/by/4.0/. The Creative Commons Public Domain Dedication waiver (http://creativecommons.org/publicdomain/zero/1. 0/) applies to the data made available in this article, unless otherwise stated in a credit line to the data. 
(Continued from previous page)

Conclusions: Children with rheumatic diseases increase in antibody titres after influenza immunization, however, it remains uncertain whether a protective level is achieved.

Keywords: Paediatric, Biological DMARDs, Immunization, Influenza, Antibody, Rheumatic

\section{Background}

Immunization is considered an effective preventive measure in reducing the risk of illness due to infection by influenza viruses. For children with juvenile idiopathic arthritis (JIA) and systemic connective tissue disorders prevention of infection is important, as both their underlying disease and often long-term use of immunosuppressive treatment increase the risk of a severe course of infection $[1,2]$. Therefore, for high risk groups such as immunocompromised individuals immunization including annual seasonal influenza vaccine is recommended $[3,4]$. The existing evidence for response to influenza vaccination in children with rheumatic diseases is primarily based on studies where patients are treated with combinations of corticosteroids and disease modifying antirheumatic drugs (DMARDs) $[5,6]$ or where only a limited number of patients are treated with biological DMARDs (bDMARDs) [7-9]. As bDMARDs have become increasingly important in the treatment during the last decades [10], we estimate that half of the 1,200 paediatric JIA patients in Denmark are treated with bDMARDs [11]. More knowledge is needed for paediatric patients with rheumatic diseases about the response to influenza vaccination in terms of achieving seroprotection and being clinically protected.

\section{Materials and Methods Objective}

The objective of this study was to compare the antibody response to influenza vaccine and laboratory confirmed influenza illness in children with rheumatic diseases and healthy controls.

\section{Study design and participants}

Participants were prospectively recruited from a regional hospital and two tertiary referral hospitals in Denmark from September 2015 to January 2016. Inclusion criteria for all participants were age 6 months to 19 years and vaccination against seasonal influenza 2015-2016. Patients were suffering from a rheumatic disease and were vaccinated due to compromised immune system assessed by the clinician given the disease itself or the medical treatment. Controls were immunologically healthy children who were not taking any medication influencing the immune system, and they were vaccinated due to a chronic illness or as sibling to a child in treatment for cancer.
The patients were grouped according to medical treatment or disease as follows:

'bDMARDs monotherapy': Patients receiving bDMARDs except Rituximab;

'bDMARDs + DMARDs': Patients receiving bDMARDs (except Rituximab) and DMARDs;

'Rituximab': Patients receiving Rituximab at any time within the last six months before vaccination and possibly additional immunosuppressive medicine;

'Systemic disorders': Patients with systemic connective tissue disorders independent of medical treatment;

'Other': Patients with rheumatic diseases (except systemic connective tissue disorders) receiving immunosuppressive medications in combinations different from previous mentioned groups.

\section{Vaccination}

Participants were vaccinated according to the guidelines for seasonal influenza from the Danish Health Authority and the Danish Paediatric Society, which recommend vaccination to risk groups $[12,13]$. Children aged 6 months to 8 years not previously vaccinated against influenza were vaccinated twice with an interval of at least 4 weeks. Children aged 9 years and above, and children previously vaccinated against influenza, were only vaccinated once. The injection was administered intramuscularly in the deltoid.

The vaccines used were: Fluarix ${ }^{\oplus}$, GlaxoSmithKline, Australia or Vaxigrip ${ }^{\oplus}$, Sanofi, France. According to the WHO recommendation for the northern hemisphere in 2015-2016 the vaccines included A/California/07/2009like (H1N1)pdm09 (A/Cal H1N1pdm09); A/Switzerland/9, 715,293/2013-like (H3N2) (A/Swi H3N2); and B/Phuket/ 3073/2013-like (Yamagata-lineage) (B/Phu Yamagata) [14].

\section{Serological testing}

The pre-vaccine blood sample was collected before vaccination or up to 3 days after vaccination [15]. The postvaccine blood sample was collected as close to day 28 as possible, but up to day 120 post vaccination, at the first following routine blood sampling. The haemagglutination inhibition (HI) assay was used to test for the presence of antibodies against the influenza virus strains contained in the vaccine. Analyses were performed at the National Influenza Center, Statens Serum Institut, Copenhagen, Denmark according to the techniques described by WHO [16]. 
A selection of samples was also tested for functional antibodies by microneutralisation ( $\mathrm{MN}$ ) assay as described by WHO [16] but with the following modifications; influenza positive cells were immunostained with 3,3'-Diaminobenzidine (DAB) (Kem-En-Tec, diagnostics 4170) followed by manual inspection in microscope.

\section{Immunogenicity}

Vaccination immunogenicity parameters were based on the European Medicines Evaluation Agency/Committee for Proprietary Medical Products (EMEA/CPMP) 1997 criteria for HI-assays [17]: Seroconversion was defined as negative pre-vaccine serum with post-vaccine serum $\geq$ 40; or a $\geq 4$ fold rise in antibody titre [17]. There is no clear definition for seroprotection in children and therefore both a titre of $\geq 40$ which is an estimate for $50 \%$ clinical protection in adults $[18,19]$, and a titre of $\geq 110$ which is an estimate for $50 \%$ clinical protection in children aged 6 months to 6 years [20] were used.

\section{Flu-like symptoms}

If the child got flu-like symptoms a questionnaire addressing clinical symptoms was to be completed and a nasal swab was performed at home. Upon inclusion the family was taught how to use the nasal swab, and test kit and a questionnaire were handed over. After each selfreported episode of flu-like symptoms the family was sent a new test kit and questionnaire.

Nasal swab was performed using Universal Transport Media kit $3 \mathrm{ml}$, UTM ${ }^{\mathrm{m}}$, COPAN. Viral RNA was extracted using $200 \mu \mathrm{L}$ of sample material and the MagNA Pure LC Total Nucleic Acid Isolation Kit on the MagNapure 96/32 (Roche). Quantitative multiplex reverse transcriptase polymerase chain reaction qRT-PCR was used for detection and subtyping of influenza A and B viruses using the MX3005P Stratagene platform. Analyses were performed at the National Influenza Center, Statens Serum Institut, Copenhagen, Denmark, using in house designed assays.

\section{Statistical analysis}

Quantitative data are presented as median values with inter quartile range (IQR) or as geometric means titre (GMT) with $95 \%$ confidence intervals $(95 \% \mathrm{CI})$. Categorical data are presented as counts (\%). To compare the groups with respect to the differences in pre-vaccine versus post-vaccine antibody titres for A/Cal H1N1pdm09, A/Swi H3N2, respectively B/Phu Yamagata, linear mixed effects models were used. The antibody levels were analysed on the log scale. The group and the timing (pre/ post) were considered fixed effects and a random intercept was included for each participant. To investigate whether the increase in antibody titres differed between the groups a test of interaction between group and timing was performed. Due to low power of this test (because of the large number of degrees of freedom), an additional test of interaction between all patients as a group/controls and timing was performed. The analyses were adjusted for the number of days between vaccination and post vaccination blood sampling as fixed effect due to an imbalance in the timing between the groups and the linearity was assessed using linear splines with knots at day 60 and 120 . To examine the effect of age (in groups 6 months to 11 years and 12-19 years), sex, duration of treatment with bDMARDs, and time from last injection of bDMARDs to vaccination, the analyses were repeated including these variables as fixed effects.

To compare the proportion of participants with seroprotection pre-vaccine to post-vaccine, the McNemar test was performed for all three influenza strains, for each group separately as well as for all patients. The analyses could not be performed when one of the proportions were $100 \%$. For each outcome and each cut off, the p-values for each group were adjusted for multiple testing using the Bonferroni method.

Data was analysed using SAS Enterprise Guide version 7.1.

\section{Results}

A total of 245 children were eligible for inclusion and 226 patients and 15 controls were enrolled (Fig. 1). Median age for patients and controls were 14.2 years and 12.2 years, respectively. Baseline characteristics are shown in Table 1 . The reasons for vaccination among
245 children vaccinated against
seasonal influenza 2015-2016
229 patients
16 controls

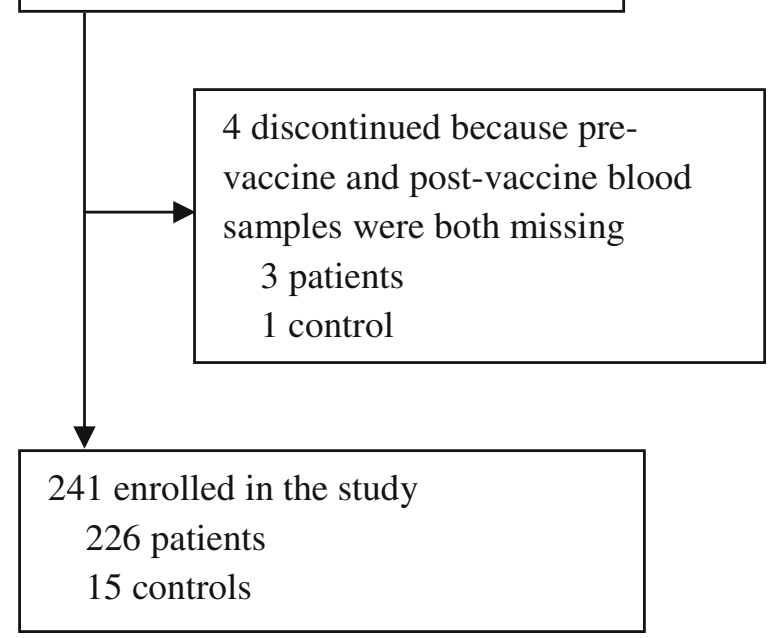

Fig. 1 Flow-chart of participants in the study 
Table 1 Characteristics of 226 patients and 15 controls vaccinated against seasonal influenza 2015-2016

\begin{tabular}{|c|c|c|c|c|c|c|c|}
\hline Characteristics & $\begin{array}{l}\text { All patients } \\
(n=226)\end{array}$ & $\begin{array}{l}\text { bDMARDs } \\
\text { monotherapy } \\
(n=80)\end{array}$ & $\begin{array}{l}\text { bDMARDs } \\
+ \text { DMARDs } \\
(n=110)\end{array}$ & $\begin{array}{l}\text { Rituximabc } \\
(n=5)\end{array}$ & $\begin{array}{l}\begin{array}{l}\text { Systemic } \\
\text { disorders } \\
(n=18)\end{array} \\
(n=1\end{array}$ & $\begin{array}{l}\text { Other }^{\mathrm{e}} \\
(n=13)\end{array}$ & $\begin{array}{l}\text { Controls } \\
(n=15)\end{array}$ \\
\hline Age, years & $14.2(10.8-17.1)$ & $14.1(10.1-17.2)$ & $13.7(11.2-16.7)$ & $15.3(14.6-16.4)$ & $15.9(12.3-16.9)$ & $17.2(14.5-18.4)$ & $12.2(11.0-15.5)$ \\
\hline Male sex & $78(34.5)$ & $30(37.5)$ & $36(32.7)$ & 0 & $6(33.3)$ & $6(46.2)$ & $9(60.0)$ \\
\hline Age at onset of symptoms & $7.8(3.2-11.0)$ & $7.1(2.9-9.5)$ & $7.0(2.8-11.0)$ & $11.3(10.1-12.5)$ & $11.0(8.8-14.3)$ & $9.6(6.3-11.5)$ & - \\
\hline Age at diagnosis & $9.5(4.4-12.8)$ & $8.0(3.4-11.9)$ & $9.5(5.3-12.7)$ & $12.8(10.1-13.4)$ & $11.9(10.0-14.8)$ & $10.3(7.8-12.2)$ & - \\
\hline \multicolumn{8}{|l|}{ JIA - ILAR criteria } \\
\hline Systemic arthritis & 7 & 3 & 2 & & & 2 & \\
\hline Oligoarthritis & 67 & 35 & 31 & & & 1 & \\
\hline Polyarthritis (RF-negative) & 81 & 27 & 50 & 1 & & 3 & \\
\hline Polyarthritis (RF-positive) & 5 & 1 & 4 & & & & \\
\hline Psoriatic arthritis & 14 & 3 & 10 & & & 1 & \\
\hline Enthesitis-related arthritis & 11 & 4 & 6 & & & 1 & \\
\hline
\end{tabular}

Undifferentiated arthritis

Systemic connective tissue disorders

Granulomatosis with polyangiitis
Juvenile dermatomyositis
Mixed connective tissue disease
Polymyositis
Scleroderma
Sjogren syndrome
Systemic lupus erythematosus
Other mixed connective tissue syndrome

Other

Behchet's disease
Chronic recurrent multifocal osteomyelitis
Familial Mediterranean fever
Iridocyclitis
Sarcoidosis

Duration of bDMARDs therapy, years

Duration from last bDMARDs injection to vaccination, days

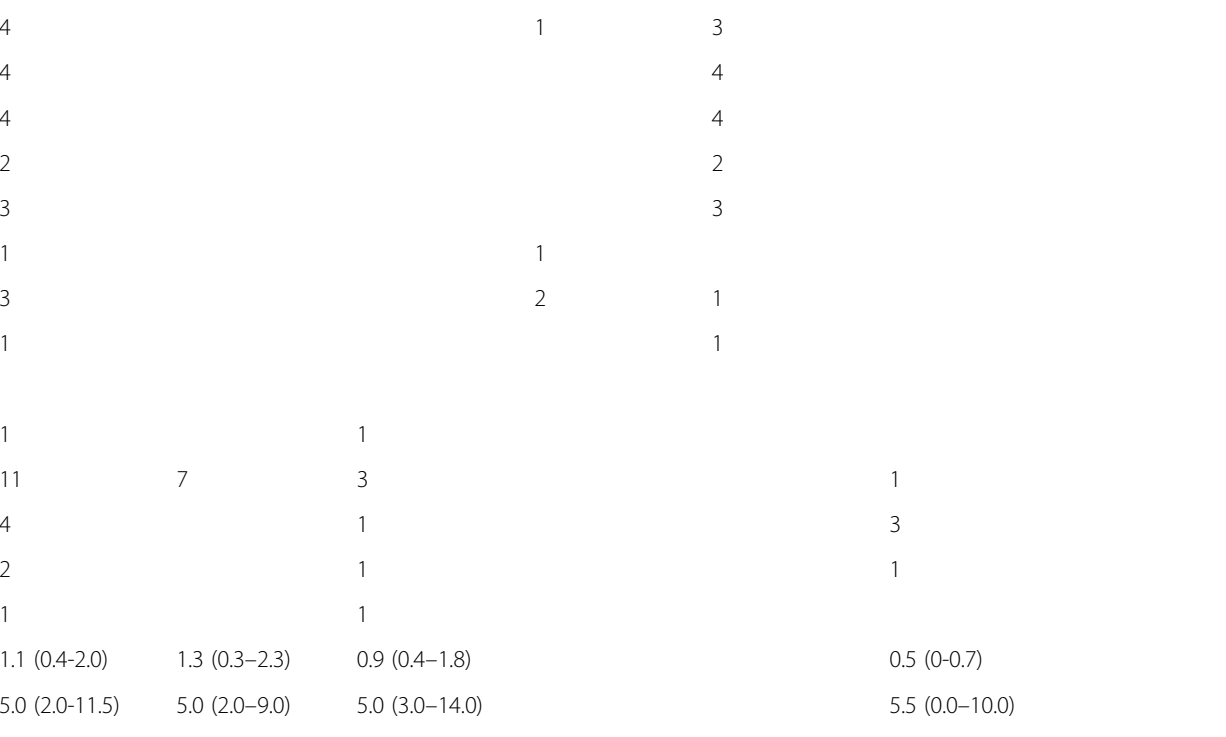

Data are median (interquartile range) or No. (\%)

Abbreviations: DMARDs disease modifying antirheumatic drugs. bDMARDs biological DMARDs. ILAR International League of Associations for Rheumatology. JIA Juvenile idiopathic arthritis. RF Rheumatoid factor

${ }^{a}$ bDMARDs used: TNFa inhibitors $87.5 \%$, costimulation modulator (inhibits T lymphocyte) 1,25\%, IL-6 inhibitor $11.25 \%$

b bDMARDs used: TNFa inhibitors $87.4 \%$, costimulation modulator (inhibits T lymphocyte) $4.5 \%$, IL-1 inhibitor $0.9 \%$, IL-6 inhibitor $8.1 \%$. DMARDS used:

Methotrexate $94.5 \%$, leflunomide $3.6 \%$, mycophenolate mofetil $0.9 \%$, colchicine $0.9 \%$, methotrexate + colchicine $0.9 \%$

c Medication in addition to rituximab: glucocorticoid + immunoglobulin + mycophenolate mofetil $20 \%$, glucocorticoid + hydroxychloroquine + mycophenolate mofetil $40 \%$, glucocorticoid + hydroxychloroquine + mycophenolate mofetil + glucocorticoid bolus $20 \%$, leflunomide $20 \%$

d Medication: glucocorticoid + mycophenolate mofetil + hydroxychloroquine $27.8 \%$, glucocorticoid + mycophenolate mofetil $11.1 \%$, glucocorticoid + immunoglobulin + methotrexate $5.6 \%$, mycophenolate mofetil $16.7 \%$, methotrexate $27.8 \%$, hydroxychloroquine $5.6 \%$, no medication $5.6 \%$

e Medication: glucocorticoid bolus + TNFa inhibitor + glucocorticoid + methotrexate $7.7 \%$, glucocorticoid bolus + IL-6 inhibitor + glucocorticoid + methotrexate

$7.7 \%$, glucocorticoid bolus + TNFa inhibitor + methotrexate $7.7 \%$, glucocorticoid bolus + TNFa inhibitor + mycophenolate mofetil $7.7 \%$, TNFa inhibitor + glucocorticoid + methotrexate $15.4 \%$, IL-6 inhibitor + glucocorticoid + methotrexate $7.7 \%$, IL-1 inhibitor + glucocorticoid $7.7 \%$, TNFa inhibitor + glucocorticoid + mycophenolate mofetil $7.7 \%$, glucocorticoid + leflunomide $7.7 \%$, no medication $23.1 \%$

controls were: Spinal muscular atrophy $(n=1)$, spherocytosis $(n=4)$, and healthy sibling to a child in treatment for cancer $(n=10)$.

The GMTs for the influenza strains contained in the 2015-2016 vaccine for patients and controls are shown in Table 2. Two patients had the pre-vaccine blood sample drawn one day after vaccination. The patients had the post-vaccine blood sample taken later (median 65 days, range 28-120) than controls (median 35 days, range 28-59) and as this 30-day difference in median time from vaccination to post-vaccine blood sampling was found to influence the level of the antibody titres, the increase in antibody level was adjusted for the number of days between vaccination and post-vaccination blood sampling. No differences were found in the increase of antibodies from pre-vaccine to post-vaccine between 


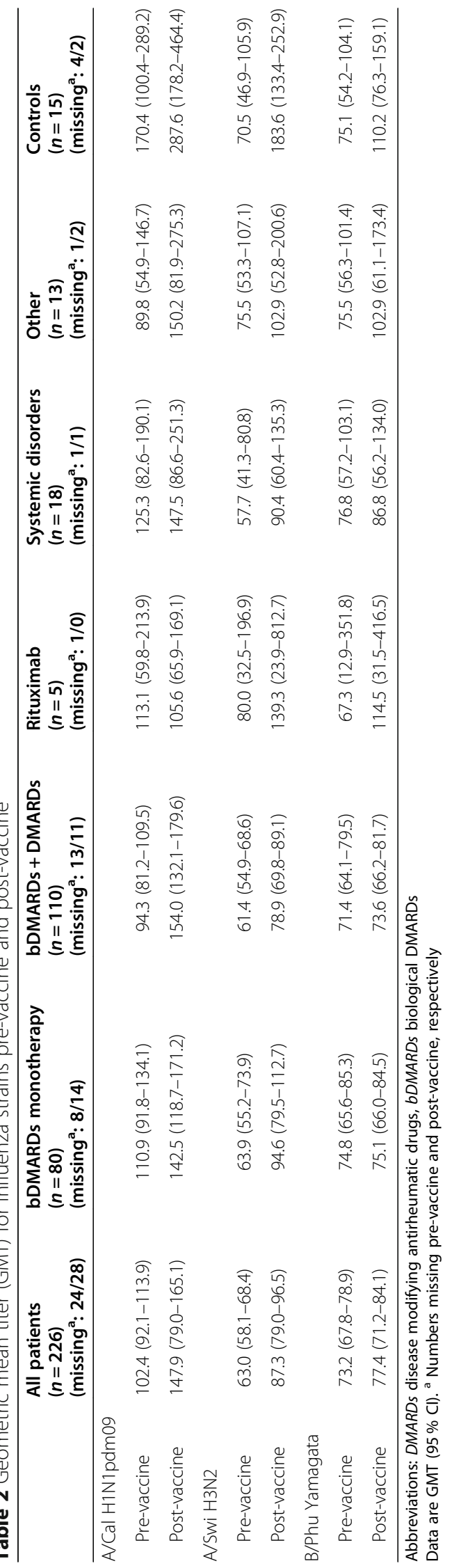


the groups: A/Cal H1N1pdm09 $(p=0.28), \mathrm{A} / \mathrm{Swi} \mathrm{H} 3 \mathrm{~N} 2$ $(p=0.15)$ and $\mathrm{B} / \mathrm{Phu}$ Yamagata $(p=0.08)$ (Table 3). Combining all the patients across groups into one group and comparing the differences between all patients and controls, we found that the percentage increase in antibodies was lower for patients compared to controls for A/Swi H3N2 with $74.9 \%$ versus $193.2 \%$ at day 28 post vaccination $(p=0.02)$, whereas no differences were found for $\mathrm{A} /$ Cal H1N1pdm09 with $62.7 \%$ versus $74.2 \%(p=0.80)$; or B/Phu Yamagata with $12.3 \%$ versus $53.9 \%(p=0.06)$ at day 28 post vaccination.

The estimated decreases in antibody levels per 30 days for the time interval $28-120$ days post vaccination were as follows: A/Cal H1N1pdm09: $-3.9 \%$ (95\% CI $-9.8-$ 1.9); A/Swi H3N2: $-7.6 \%$ (95\% CI -12.3- -2.9); and B/ Phu Yamagata: -1.0 \% (95\% CI -4.8-2.8).

For all three influenza strains no associations were found between the increase in antibody level after vaccination and age, sex, duration of bDMARDs treatment, or time from injection of bDMARDs to vaccination (results not shown).

Seroprotection was evaluated for both pre-vaccine and post-vaccine samples at the cut offs for antibody titres of $\geq$ 40 and $\geq 110$ (Table 4). Among all patients the pre-vaccine rates for seroprotection $\geq 40$ were $93.1-97.0 \%$ for all three strains. An increase in the proportions of patients being seroprotected after vaccination was found for $\mathrm{A} / \mathrm{Swi}$ H3N2 whereas no increases were seen for A/Cal H1N1pdm09 and $\mathrm{B} / \mathrm{Phu}$ Yamagata. For seroprotection $\geq 110$ the prevaccine rates for all patients were $14.9 \%-43.6 \%$ and for $\mathrm{A} / \mathrm{Cal} \mathrm{H} 1 \mathrm{~N} 1 \mathrm{pdm} 09$ and $\mathrm{A} / \mathrm{Swi} \mathrm{H} 3 \mathrm{~N} 2$ this rose to $65.2 \%$ and $31.3 \%$ post-vaccine (both $p \leq 0.0001$ ), respectively while there was no increase for B/Phu Yamagata.

The MN assay was used for reanalysing a subset of the blood samples (11 patients and 9 controls) to investigate the performance of the antibodies in this functional assay. The results showed that participants having an increase in antibody titres in the HI assay also increased with the MN assay (Fig. 2). Furthermore, we found that more participants increased in the $\mathrm{MN}$ assay compared to the HI assay.

Flu-like illness was registered in 42 patients of whom 5 experienced 2 episodes, compared to no episodes among controls. The frequencies of symptoms registered in $96 \%$ of the episodes: Malaise $87 \%$, sore throat $76 \%$, head ache $76 \%$, fever $73 \%$, cough $63 \%$, sore muscles $53 \%$, and stomach ache $44 \%$. None of the patients were tested positive for any of the influenza strains included in the vaccine but $12(26 \%)$ were tested positive for influenza B Victoria.

\section{Discussion}

In this prospective multicentre cohort study, we investigated the response to vaccination against seasonal influenza 2015-2016 in children with rheumatic diseases and immunologically healthy controls; all patient groups and controls increased in antibody titres after vaccination.

The pre-vaccine GMTs for A/Swi H3N2 and B/Phu Yamagata were about 70 for both patients and controls, whereas for A/Cal H1N1pdm09 pre-vaccine GMT was about 100 for patients and 170 for controls. In this study we had no information about previous vaccinations against influenza but due to medical treatment and disease some children had been vaccinated before. Previous infections by circulating virus strains could affect these titres, however A/Cal H1N1pdm09 had been included in the seasonal vaccine in the years preceding our study and this could explain the higher pre-vaccine titre against this strain [14]. In contrast, A/Swi H3N2 and B/Phu Yamagata were both introduced in the 2015-2016 vaccine and this may explain the lower pre-vaccine titres [21].

No differences in the percentage increase in antibody titres after vaccination between patient groups and controls were found. However, when combining all the patients across groups into one group and comparing antibody increase between these and controls we found a lower response for patients to A/Swi H3N2, and a tendency to a lower response for $\mathrm{B} / \mathrm{Phu}$ Yamagata. It is possible that we would have found a difference in the initial comparisons between groups if the number of participants in each group had been larger.

More than $90 \%$ of patients and controls were already protected pre-vaccine against the three influenza strains using the cut-off for seroprotection $\geq 40$. Consequently, the proportion of patients reaching this protective level after vaccination was only significant for A/Swi H3N2.

The proportion of children protected pre-vaccine was substantially lower using the cut-off for seroprotection $\geq$ 110 and only few groups had a significant proportion of patients achieving this level after vaccination. Since most participants in our study were older than 6 years and no clearly defined cut off for seroprotection in children above 6 years exists, it is therefore uncertain whether we should assume that the patients were mainly protected or unprotected. A likely explanation for patients not reaching seroprotection $\geq 110$ could be immunosuppression as we found that for all three strains the percentages of controls protected after vaccination were higher for controls than patients. However, the crude numbers for antibody titres were used for estimation of seroprotection and direct comparisons between groups were not performed. As the post-vaccine blood samples were taken later for patients than controls, and antibodies were shown to decline beyond day 28 post vaccination, some patients might have been misclassified as being unprotected compared to controls who had the postvaccine blood sample taken earlier. 


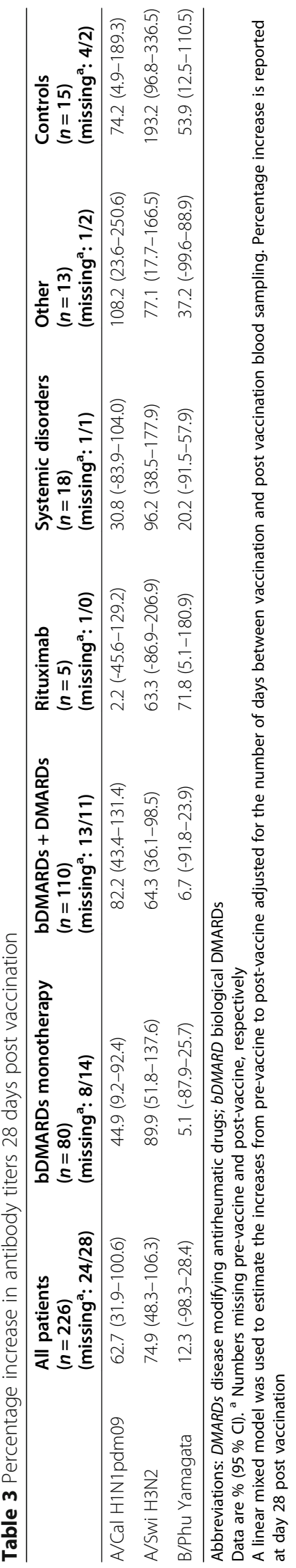




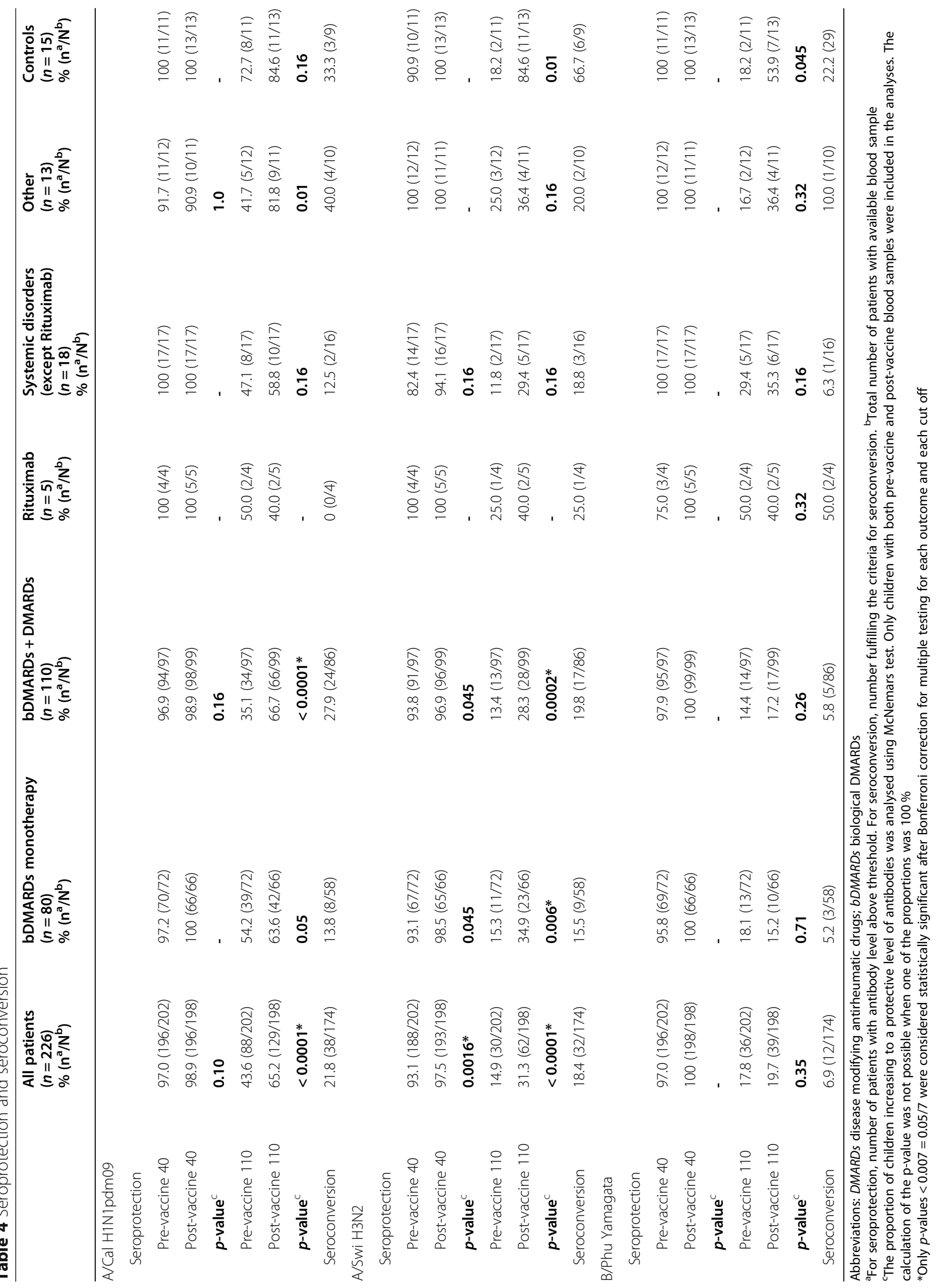




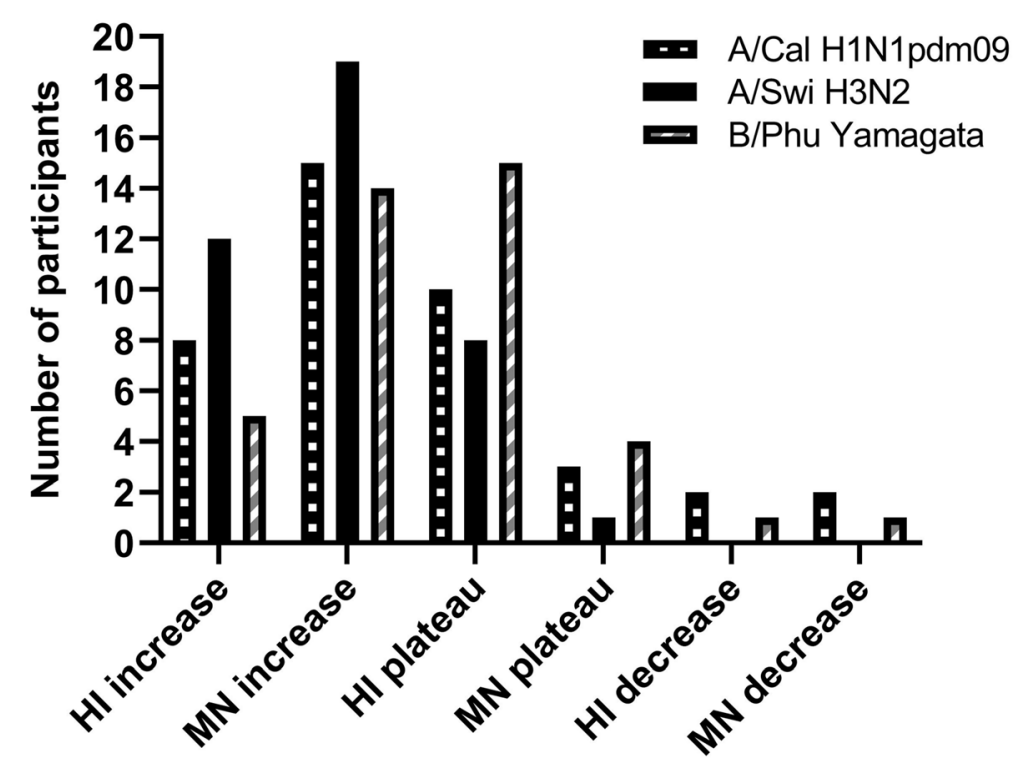

Fig. 2 Response to vaccination using haemagglutination inhibition assay and microneutralisation assay . Abbreviations: HI: haemagglutination inhibition; MN: microneutralisation. A selection of samples was both tested for the presence of antibodies using the HI assay and for functional antibodies by MN. From the pre-vaccine sample to the post-vaccine sample the antibody titre was shown to increase, decrease or remain at a plateau

Previous studies in children with rheumatic diseases have focused on the seroprotection $\geq 40$ cut off $[5,7,8]$. As our results showed that the proportions of children being protected after vaccination were very different depending on which cut off was used, it emphasizes the need for defining a better correlate for protection in children above 6 years in future clinical studies.

We investigated the differences in antibody response depending on immunosuppression, and the lowest increase was expected for the patients receiving Rituximab. But as described above, no differences were found between the patient groups, and even when looking at the percentage increase in antibody titres and proportions of patients achieving seroprotection, there were not a clear tendency for any group to have a better or worse response to all three influenza strains than the remaining groups.

Previous studies of response to seasonal influenza vaccine among patients with rheumatic diseases are not comparable due to differences in rheumatic disease and/ or laboratory method and/or influenza virus strain but the overall findings are in line with our results [6-8].

As our results showed a limited increase in antibody titres, we chose to analyse the functionality of antibodies in a sub-set of samples using the MN assay. We found that participants having increasing antibody titres using the $\mathrm{HI}$ assay also increased using the $\mathrm{MN}$ assay. Furthermore, a higher number of participants had increasing antibody titres using the MN assay compared to the HI assay indicating that the $\mathrm{MN}$ assay was more sensitive.
This is in line with previous findings [22] and it strengthens the assumption that our results from the HI assays are a reliable correlate for antibody response to the influenza vaccine in our cohort.

The population were primarily infected by $\mathrm{A} / \mathrm{Cal}$ H1N1pdm09 (51\%), or influenza B Victoria (44\%) in the 2015-2016 influenza season in Denmark [21]. The influenza B Victoria strain was not included in the vaccine [21]. Since patients with influenza-like illness only tested positive for influenza B Victoria, it was not possible to investigate the relation between antibody levels and clinical infection caused by the three strains included in the vaccine. The influenza A/Cal H1N1pdm09 strain was circulating but no clinical cases were seen in our cohort possible due to protective antibody levels.

A strength for this study is the prospectively inclusion from three geographical different centres, and we assume that it is representative for paediatric patients with rheumatic disease who are vaccinated against seasonal influenza due to immunosuppression. About one third of all Danish JIA patients treated with bDMARDs participated in this study, thereby contributing with important knowledge from a larger group of patients compared to previous studies [7-9].

The study also has some limitations. Only a minor number of controls were included and thus we were not able to conduct the intended gender and age-matched study design. We intended to include 139 patients and 69 controls for comparisons, but this was not achieved due to difficulties in recruiting and it limits our ability to 
find a difference between the groups.For the completed participants $20 \%$ had a missing blood sample either prevaccine or post-vaccine. The reason for missing samples was not registered but we assume that they were missing at random. Therefore, we chose a statistical model where all patients contributed even when either the pre-vaccine or post-vaccine sample was missing. Furthermore, the patients in our study had the post-vaccine blood sample taken later than controls, and due to this imbalance, we adjusted for days from vaccination to post-vaccine blood sampling in the analyses. Lastly, we grouped the patients based on medical treatment or disease, but the results are weak due to small numbers.

\section{Conclusions}

In conclusion, children with rheumatic disease and healthy controls increase in antibody titres after influenza immunization. Depending on the cut-off for seroprotection it remains, nevertheless, unclear whether a protective level is achieved. However, none of the patients and healthy controls were tested positive for any of the influenza strains included in the vaccine. The results suggest that influenza vaccination provides protection in immunocompromised children with rheumatic disease, although possibly less so than in healthy children.

Our findings emphasize the need for defining a better correlate for protection in children in future clinical studies and lastly, time from vaccination to post-vaccine blood sampling was found to be an important confounder so future studies should take this into account.

\section{Abbreviations}

A/Cal H1N1pdm09: A/California/07/2009-like (H1N1)pdm09; A/Swi H3N2: A/ Switzerland/9715293/2013-like (H3N2); B/Phu Yamagata: B/Phuket/3073/ 2013-like (Yamagata-lineage); bDMARDs: Biological disease modifying antirheumatic drugs; DMARDs: Disease modifying antirheumatic drugs; GMT: Geometric means titre; HI: Haemagglutination inhibition; JIA: Juvenile idiopathic arthritis; MN: Microneutralisation

\section{Acknowledgements \\ We thank all the patients, controls and their parents for their consent to participate in this study. We thank the nurses, secretaries and medical doctors at the paediatric departments at Rigshospitalet, Naestved Hospital and Odense University Hospital for extensive help and collaboration. Thanks to laboratory technicians at Rigshospitalet, Odense University Hospital and Naestved Hospital for taking and handling the blood samples. Laboratory technicians at Statens Serum Institut is thanked for performing the HI assays, MN assays, and influenza virus qRT-PCR diagnostics.}

\section{Authors' contributions}

The scientific problem was identified in collaboration between $L J, S N, A E C$, FKP and AP. $\amalg$ wrote the study protocol. The planning for each study site and inclusion was performed in collaboration between $L, S N, A E C, P T, A B$ and PSW. $\amalg$ collected the data in the database. RT and TKF were responsible for the assays and PCR diagnostics for influenza. The statistical analyses were planned and performed by $L J$ and SR. $L J$ wrote the first draft of the manuscript. All authors have revised the manuscript, and all have read and approved the final manuscript.

\section{Funding}

The study was supported by the Rigshospitalet Research Foundation, Copenhagen University Hospital; the Danish Rheumatism Association; the Foundation for Collaboration between Rigshospitalet and Odense University Hospital; the Dagmar Marshall Foundation and the KV Foundation. The funding sources had no influence on any part of this study.

\section{Availability of data and materials}

The datasets generated and analysed during the current study are not publicly available. Consent for making the datasets publicly available was not obtained.

\section{Declarations}

Ethics approval and consent to participate

The regional scientific ethical committee for the Capital Region of Denmark approved the study (H-I-2014-095). Informed consent was obtained from participants and parents as relevant according to the age of the participant.

Consent for publication

Not applicable.

\section{Competing interests}

The authors declare that they have no competing interests.

\section{Author details}

${ }^{1}$ The Department of Paediatrics and Adolescent Medicine, Juliane Marie Centre, Rigshospitalet, Copenhagen University Hospital, Copenhagen, Denmark. ${ }^{2}$ Faculty of Health and Medical Sciences, University of Copenhagen, Copenhagen, Denmark. ${ }^{3}$ The Department of Paediatrics and Adolescent Medicine, Hans Christian Andersen Children's Hospital, Odense University Hospital, Odense, Denmark. ${ }^{4}$ Department of Virus and Microbiological Special diagnostics, National Influenza Center, Statens Serum Institut, Copenhagen, Denmark. ${ }^{5}$ Section of Biostatistics, Department of Public Health, University of Copenhagen, Copenhagen, Denmark. ${ }^{6}$ The Department of Paediatrics, Naestved Hospital, Naestved, Denmark.

Received: 22 April 2020 Accepted: 4 March 2021

Published online: 12 March 2021

\section{References}

1. Beukelman T, Xie F, Chen L, Baddley JW, Delzell E, Grijalva CG, et al. Rates of hospitalized bacterial infection associated with juvenile idiopathic arthritis and its treatment. Arthritis \& Rheumatism. 2012 Aug 1;64(8):2773-80.

2. Singh JA, Wells GA, Christensen R, Ghogomu ET, Maxwell LJ, MacDonald JK, et al. Adverse effects of biologics: a network meta-analysis and Cochrane overview. Cochrane Database of Systematic Reviews [Internet]. 2011 [cited 2018 Sep 17];(2). Available from: https://www.cochranelibrary.com/cdsr/ doi/10.1002/14651858.CD008794.pub2/abstract

3. Heijstek MW, Bruin LMO de, Bijl M, Borrow R, Klis F, van der, Koné-Paut I, et al. EULAR recommendations for vaccination in paediatric patients with rheumatic diseases. Ann Rheum Dis. 2011 Jan 10;70(10):1704-12.

4. Pinto MV, Bihari S, Snape MD. Immunisation of the immunocompromised child. J Infect. 2016;72:13-22.

5. Kanakoudi-Tsakalidou F, Trachana M, Pratsidou-Gertsi P, Tsitsami E, Kyriazopoulou-Dalaina V. Influenza vaccination in children with chronic rheumatic diseases and long-term immunosuppressive therapy. Clin Exp Rheumatol. 2001;19(5):589-94.

6. Ogimi C, Tanaka R, Saitoh A, Oh-ishi T. Immunogenicity of Influenza Vaccine in Children With Pediatric Rheumatic Diseases Receiving Immunosuppressive Agents: The Pediatric Infectious Disease Journal. 2011; 30(3):208-11.

7. Woerner A, Sauvain M-J, Aebi C, Otth M, Bolt IB. Immune response to influenza vaccination in children treated with methotrexate or/and tumor necrosis factor-a inhibitors. Human Vaccines. 2011 Dec 1;7(12):1293-8

8. Shinoki T, Hara R, Kaneko U, Miyamae T, Imagawa T, Mori M, et al. Safety and response to influenza vaccine in patients with systemic-onset juvenile idiopathic arthritis receiving tocilizumab. Mod Rheumatol. 2012;22(6):871-6.

9. Camacho-Lovillo MS, Bulnes-Ramos A, Goycochea-Valdivia W, FernándezSilveira L, Núñez-Cuadros E, Neth $O$, et al. Immunogenicity and safety of influenza vaccination in patients with juvenile idiopathic arthritis on 
biological therapy using the microneutralization assay. Pediatr Rheumatol Online J [Internet]. 2017 Aug 7;15. Available from: https:/www.ncbi.nlm.nih. gov/pmc/articles/PMC5547451/.

10. Stoll ML, Cron RQ. Treatment of juvenile idiopathic arthritis: a revolution in care. Pediatr Rheumatol Online J. 2014;12:13.

11. Børnegigt. - årsager, symptomer og behandling [Internet]. The Danish Rheumatism Association. [cited 2019 Jan 31]. Available from: https://www. gigtforeningen.dk/viden-om-gigt/diagnoser/boernegigt/.

12. Bekendtgørelse om gratis influenzavaccination til. visse persongrupper retsinformation.dk [Internet]. [cited 2018 Jun 19]. Available from: https:// www.retsinformation.dk/eli/lta/2015/1067.

13. Infektionsmedicin | Selskabet [Internet]. [cited 2018 Jun 19]. Available from: http://www.paediatri.dk/infektionsmedicin-vejl.

14. WHO | Recommended composition of influenza. virus vaccines for use in the 2015-2016 northern hemisphere influenza season [Internet]. WHO. [cited 2018 Apr 20]. Available from: http://www.who.int/entity/influenza/va ccines/virus/recommendations/2015_16_north/en/index.html.

15. Cox RJ, Brokstad KA, Zuckerman MA, Wood JM, Haaheim LR, Oxford JS. An early humoral immune response in peripheral blood following parenteral inactivated influenza vaccination. Vaccine. 1994;12(11):993-9.

16. World Health Organization, editor. Manual for the laboratory diagnosis and virological surveillance of influenza. Geneva: World Health Organization; 2011. $139 \mathrm{p}$.

17. The European Agency for the Evaluation of Medicinal Products (EMEA). Comitte for Proprietary Medicinal Products (CPMP). Note for guidence on harmonisation of requirements for influenza vaccines. [Internet]. . [cited 2017 Oct 27]. Available from:http://www.ema.europa.eu/docs/en_GB/ document_library/Scientific_quideline/2009/09/WC500003945.pdf.

18. Hobson D, Curry RL, Beare AS, Ward-Gardner A. The role of serum haemagglutination-inhibiting antibody in protection against challenge infection with influenza A2 and B viruses. J Hyg (Lond). 1972;70(4):767-77.

19. Davies JR, Grilli EA. Natural or vaccine-induced antibody as a predictor of immunity in the face of natural challenge with influenza viruses. Epidemiol Infect. 1989;102(2):325-33.

20. Black S, Nicolay U, Vesikari T, Knuf M, Giudice GD, Cioppa GD, et al. Hemagglutination Inhibition Antibody Titers as a Correlate of Protection for Inactivated Influenza Vaccines in Children. The Pediatric Infectious Disease Journal. 2011;30(12):1081-5.

21. Krause TG, Knudsen LK, Emborg H-D, Nielsen J, Espenahin L, Ricotta L, et al. Uge 24-2016 Influenzasæsonen 2015/2016 [Internet]. Statens Serum Institut. 2016 [cited 2018 Dec 12]. Available from: https:/www.ssi.dk/aktuelt/ nyhedsbreve/epi-nyt/2016/uge-24---2016.

22. Verschoor CP, Singh P, Russell ML, Bowdish DME, Brewer A, Cyr L, et al. Microneutralization Assay Titres Correlate with Protection against Seasonal Influenza H1N1 and H3N2 in Children. PLoS One [Internet]. 2015 Jun 24 [cited 2018 Oct 25];10(6). Available from: https://www.ncbi.nlm.nih.gov/ pmc/articles/PMC4479562/.

\section{Publisher's Note}

Springer Nature remains neutral with regard to jurisdictional claims in published maps and institutional affiliations.

Ready to submit your research? Choose BMC and benefit from:

- fast, convenient online submission

- thorough peer review by experienced researchers in your field

- rapid publication on acceptance

- support for research data, including large and complex data types

- gold Open Access which fosters wider collaboration and increased citations

- maximum visibility for your research: over $100 \mathrm{M}$ website views per year

At $\mathrm{BMC}$, research is always in progress.

Learn more biomedcentral.com/submissions 